\title{
DE LA ORGANIZACION DEL PIRINEO EN EL PASADO A LA ORDENACION DE SU TERRITORIO EN EL FUTURO
}

\author{
Vicente BIELZA DE ORY
}

\author{
Dpto. de Geografía y Ordenación del Territorio \\ Universidad de Zaragoza
}

\begin{abstract}
Resumen: El espacio pirenaico a lo largo de la historia ha sido afectado por dos tipos de cambio: en su organización político-territorial y en su funcionalidad socioeconómica. Desde el punto de vista político los grandes hitos se corresponden con la relativa integración del espacio pirenaico hasta la constitución de los Estados modernos, su desintegración y vida de espaldas a partir del robustecimiento de las fronteras y un tercer hito con la configuración del Mercado Unico Europeo y la actual supresión de fronteras. En el curso del tiempo también ha cambiado el Pirineo de ser un espacio productivo primario en la era preindustrial a introducirse algunas actividades secundarias y a ser afectado por todos los procesos generales externos de industrializaciónurbanización hasta llegar a la era postindustrial en que el Pirineo se inserta como espacio verde y blanco en la civilización urbana del ocio. La ordenación del territorio pirenaico debe articularse en función de las nuevas condiciones políticas y socioeconómicas, de forma que permita disminuir los desequilibrios socioeconómicos N-S y extremos-centro de la cadena.
\end{abstract}

Palabras clave: Ordenación del Territorio, Pirineo.

Abstract: The Pyrenees space has been affected by two kind of changes along history: in its political point of view the big moments correspond to the pyrenees space relative e integration until the constitution of the modern states; its disintegration and life backwards since the strengthening of borders; and a third momento to the shops of the European single market and the present borders suppression. Throughout time the Pyrenees have also changed from being a primary productive space at the preindustrial Era to introducing secondary activities, and to being affected by all the general external processes of industrialisation urbanisation as late as the postindustriel Era when the Pyrenees territorial arrangement must be articulated following the new political, socioeconomical conditions so that the socioeconomic North-South and Centreends of the chain unbalances diminish, as well as the vertical unbalances in accordance with the new borderless mountain vocation with a main turistic function. 
Key words: Land management, Pyrenees.

\section{DISTINCION ENTRE ORGANIZACION POLITICO-TERRITORIAL Y ORDENACION DEL TERRITORIO}

Previamente al desarrollo del tema propuesto deben distinguirse dos conceptos: la organización y división del territorio desde el punto de vista políticoadministrativo y la ordenación del territorio que según la Carta Europea de Ordenación del Territorio de 1982/83 tiene como objetivos "un desarrollo equilibrado de las regiones y la organización física del espacio según un concepto rector", es decir, equilibrar horizontal y verticalmente el territorio. La política territorial tiende a "reducir las desigualdades regionales", -que en el caso del Pirineo se producen tanto en dirección N-S, entre las dos vertientes, como en sentido E-W- "y alcanzar una mejor concepción general de la utilización y organización del espacio, de la protección del medio ambiente y de la calidad de vida".

Es evidente que la división y organización político-administrativa tiene su incidencia tanto en el desarrollo equilibrado de las regiones o comarcas humanas dentro de un territorio físicamente homogéneo, cual es el pirenaico, y en menor medida en la organización física del espacio según un concepto rector. Por ello, para enfocar la política territorial actual y de futuro hay que contar con la división y organización político-administrativa del pasado y del presente, planteando la gestión del territorio en función de la propia organización de cada momento histórico, así como del estadio tecnológico de la actividad económica (preindustrial, industrial o postindustrial).

\section{EL ESPACIO INTEGRADO PIRENAICO ANTES DE LOS ESTADOS MODERNOS}

Hasta la constitución de los Estados modernos el espacio pirenaico era un espacio en el que la homogeneidad natural se traducía en una cierta homogeneidad socioeconómica que nos permite hablar de un espacio integrado $\sin$ fronteras. El alejamiento de los grandes centros de poder, como Córdoba, permite en la Edad Media el nacimiento de reinos cristianos pirenaicos, que en los extremos más accesibles del Pirineo se extienden a ambas vertientes. Por encima de la línea de cumbres había un aprovechamiento en común de los recursos naturales, tipificado ${ }^{1}$ en la figura de las

\footnotetext{
${ }^{1}$ Los tratados de "Lies et passeries" aparecen por escrito a partir del siglo XIII (7-VII-1277 entre el Valle d'Ossau y el de Canfranc, Arch. Dep. Pyrénées-Atlantiques V. d'Ossau AA2 ( $\left.F^{\circ} 23-25\right)$ ). Anteriormente, según Tucoo-Chalo, existían verbalmente.
} 
facerias (Cavailles, 1950 y Fairen Guillen, 1961-62). Existía una movilidad comercial que se materializaba en las ventas de trigo aragonés al Bearn y en las ferias anuales o semestrales a las que concurrían campesinos con sus ganados de la otra vertiente del Pirineo. Las reuniones comerciales de ferias y mercados lo eran también sociales, cristalizando en amistades e incluso en matrimonios entre individuos de uno y otro lado de la divisoria de aguas. Había también una movilidad laboral de caracter temporal y en determinados momentos migraciones definitivas de cierta entidad (la reconquista cristiano-medieval del Valle del Ebro y las guerras de religión en Francia significaron la ubicación de gentes del Pirineo septentrional en el sur; la expulsión de los moriscos de Aragón en 1610, que afectó al 15\%, de su población se compensó con gentes ultrapirenaicas). Finalmente había también una movilidad cultural intrapirenaíca que nos permite hablar de una cultura pirenaica.

\section{LA DESINTEGRACION DEL ESPACIO CON LA CONSTITUCION DE LOS ESTADOS MODERNOS}

La política de fronteras hispano-francesa iniciada por el tratado de Pirineos de 1659 y endurecida con los nacionalismos durante el siglo XIX, que se tradujo en la subida de las fronteras hacia la linea de cumbres (1841) y en el tratado de límites de 1862 propició la desintegración del espacio pirenaico y la vida de espaldas ${ }^{2}$. En el siglo XIX la revolución industrial y de los transportes abre una etapa en la que los extremos pirenaicos casi monopolizan las relaciones de largo alcance, mientras en el Pirineo Central, más inexpugnable físicamente y menos atractivo para el trazado de los circuitos de la era industrial, se mantienen las relaciones de corto alcance con una cultura pirenaica en la que siguen teniendo vigencia ancestrales figuras como la de la "casa" y el heredero único, la facería, el aviso a los vecinos en caso de guerra. Los campesinos se consideran antes montañeses pirenaicos que franceses o españoles. El contrabando era una forma de vida más allá de las fronteras político-económicas. La caza furtiva con desconocimiento de las rayas trazadas desde Paris y Madrid, una práctica habitual en la que participaban los propios alcaldes. La más pronta incorporación a la vida industrial del Norte propicia migraciones temporales o definitivas $\mathrm{S}-\mathrm{N}^{3}$. En conjunto se puede afirmar que el Pirineo Central desde el siglo

${ }^{2}$ Hacia 1800 el $47 \%$ del comercio de Basses-Pyrénées se hacía con la vertiente española, sin embargo, los cambios comerciales se habían reducido considerablemente respecto del siglo XVI y limitado al ámbito interregional (C. Desplat: "Exposición Bearn-Aragón-Navarra" (1985, p. 29).

${ }^{3}$ Las migraciones siguieron siendo hasta el siglo XIX más intensas hacia el sur, entonces relativamente más rico. Interesante fue la acogida en el Sur de los pretres réfractaires a la Revolución 1792. 
XIX supone una mayor barrerá para las relaciones de largo alcance que para las intrapirenaicas N-S (Bielza y Dalla-Rossa, 1988).

\section{LOS DESEQUILIBRIOS HORIZONTALES PROVOCADOS POR EL CENTRALISMO Y LA REVOLUCION INDUSTRIAL}

La influencia de esta desintegración, propiciada desde Madrid y Paris, en la gestión del territorio es mayor en aquellos aspectos incorporados al mismo por la revolución industrial (infraestructuras del transporte, industria, urbanización) que en el sistema rural. Aún así los desequilibrios territoriales intrapirenaicos del propio mundo rural, se acentúan en esta etapa. Max Daumas (1976) en su gran tesis doctoral puso de manifiesto el desfase N-S y entre los extremos de la cadena y el centro en los procesos del éxodo rural y de la mecanización y modernización del campo. Desfases que llegan a superar el medio siglo. Pero estos desfases en favor del $\mathrm{N}$ y de los extremos de la cadena tuvieron mucho que ver con la muy anterior llegada a estos espacios del ferrocarril y de las carreteras modernas ${ }^{4}$, siempre trazados en función de las estrategias de Paris y Madrid y no de los intereses pirenaicos. Lo mismo cabe decir de la propia industrialización: las centrales hidroeléctricas, que muchas veces exigieron la construcción de las carreteras que pusieron en comunicación los altos valles pirenaicos con el resto del Estado, fueron punto de partida para algunos focos industriales intrapirenaicos, siempre más retrasados en el tiempo en el Sur y en el centro de la cadena.

Las ciudades intrapirenaicas hasta la revolución industrial contaban con una población y un desarrollo urbano acordes con sus funciones comerciales y de servicio normalmente de alcance comarcal. La mejor accesibilidad en función de las nuevas infraestructuras del transporte y de la implantación de la industria generó el desarrollo de algunas -especialmente en el norte y en los extremos- frente al estancamiento de la mayoría. Pero lo más importante es que las redes de pequeñas ciudades intrapirenaicas se comunicaban a través de las ciudades del piedemonte o de las depresiones del Ebro y Aquitania, dándose la espalda, salvo en los extremos pirenaicos. Ello se traducía en las consiguientes deseconomías fronterizas y disimetrías en las áreas de influencia socioeconómicas y culturales de las pequeñas ciudades fronterizas.

\footnotetext{
${ }^{4} \mathrm{El}$ sistema radial de caminos poryectado por Ward dió salida por los extremos pirenaicos: Irún en 1783 y La Junquera en 1792 . El Somport se abriría en 1877 y eso que en 1764 estaba ya trazada la ruta Pau-Urdos (el miedo a la invasión retrasó la parte española). El Portalet se inauguró en 1909, Bielsa en 1976. El trazado ferroviario por Irun se inauguró en 1864, Portbou en 1878, Canfranc en 1929.
} 


\section{LOS DESEQUILIBRIOS VERTICALES EN LA ERA PREINDUSTRIAL}

Los desequilibrios verticales en el territorio pirenaico por una actividad económica inadecuada de la población sobre el medio natural se inician en la era preindustrial y afectan principalmente al bosque en determinados momentos históricos. La invasión musulmana provocó una sobrepresión demográfica de los cristianos en los valles pirenaicos, que como apunta Chauvelier (1987) eran grandes consumidores de bosque, iniciando una deforestación en los Pirineos meridionales. En los siglos XVIII y XIX, todavía con sociedades preindustriales, se llegó a la saturación demográfica en el Pirineo, provocando la puesta en cultivo, de forma itinerante con "artigueo" o de forma definitiva de suelos marginales, en pendiente, mediante bancales, a costa del bosque. La actividad pastoril, la extracción de madera para la Armada, la fabricación de carbón vegetal, las necesidades de las serrerías hicieron retroceder el bosque, de tal forma, que a fines del XVIII provocaron la alarma de expertos como Ignacio de Asso (1798). Las desamortizaciones decimonónicas privatizaron grandes extensiones de bosque público que fueron objeto de especulación y explotación desorbitada, talándolos y poniendo en cultivo muchas tierras en pendiente.

El abandono de parcelas, primero por el agotamiento de las mismas y después por el éxodo rural, provocó masivas pérdidas de suelo por erosión, desequilibrios hidrológicos graves y amenazas de aludes para las poblaciones.

\section{LOS DESEQUILIBRIOS VERTICALES EN LA ERA INDUSTRIAL}

El impacto de la industrialización en el mundo rural pirenaico agrava los desequilibrios en el ecosistema o agrosistema preindustrial. Desde el Neolítico -como ha señalado Montserrat Recoder- se produce en los pastos un equilibrio con la carga ganadera que al descender con la crisis de la trashumancia -por falta de pastores o rentabilidad- se altera con el consiguiente embastecimiento de los pastos, invasión de matorrales e incluso rearborización desordenada. Las consecuencias negativas se dan en dos sentidos, depauperación del recurso pastos e incremento de las posibilidades de incendio en los bosques.

La industrialización que aportó maquinaria a la actividad agrícola pirenaica permitió hasta mitad del siglo XX seguir ampliando el "ager" a costa del "saltus", cultivando suelos hasta entonces incultivables, haciendo en general labores más profundas y agresivas. La despoblación posterior y el consiguiente abandono de estas tierras marginales facilitó los procesos erosivos y los desequilibrios hidrológicos (Ayerbe, 1908). 
El patrimonio etnológico y cultural se desprotege al abandonarse pueblos enteros, bien por un éxodo espontáneo o bien por actuaciones de la administración al repoblar forestalmente, acotando pastos y obligando al abandono de tierras, o al construir pantanos e inundar suelo agrícola (Garcia Ruiz, 1976).

La industria contaminante de suelos y aguas plantea incompatibilidades en el uso del suelo con la agricultura o los propios núcleos de población.

Otro tipo de agresión en la era industrial se plantea con la apertura de las grandes vías de comunicación. Aunque hay casos, como al construirse la vía de Canfranc a principios del siglo $X X$, en que se protegieron y reforestaron pendientes para evitar aludes que se daban por las talas anteriores (Estebanez, 1968).

Todos estos hechos, y otros bien conocidos, dejan la montaña pirenaica al final de la era industrial en una situación de suma fragilidad.

\section{LA CEE Y LA DIVISION AUTONOMICA: NUEVOS DESEQUILIBRIOS HORIZONTALES}

La pertenencia de Francia a la CEE supuso a partir de 1960 una política de montaña que desconoció la parte española hasta 1982 (fecha en que entró en vigor la Ley de Agricultura de Montaña). Durante más de veinte años las actuaciones comunitarias ampliaron los desequilibrios norte-sur.

A partir de 1980 para preparar la vertiente pirenaica francesa al ingreso de España en la CEE se puso en marcha el "Plan du Grand Sud-Ouest" ampliado hasta 1986-93 con tres programas integrados mediterráneos (PIM) para Aquitania, MidiPyrenées y Languedoc-Rousellon. Además hay que citar un programa nacional de interés comunitario (PNIC) de cuyas actuaciones se benefician zonas pirenaicas, una operación integrada de desarrollo (OID) para el departamento de Ariège (1986-90) y un programa operativo (1992-93) en la zona de Lacq-Ortez de apoyo a la reconversión. industrial (Gorria, 1993).

A partir de la Constitución española de 1978 se propicia que cada Comunidad Autónoma pirenaica (Pais Vasco, Navarra, Aragón, Cataluña) plantee su particular política de desarrollo y ordenación.

Teniendo en cuenta además que Pais Vasco y Cataluña disponen de más competencias que Navarra, y sobre todo que Aragón, la tendencia es que se acentúen los desequilibrios en favor de los extremos pirenaicos en la vertiente española. 


\section{LA NUEVA VOCACION DE LA MONTAÑA: LOS NUEVOS DESEQUILIBRIOS VERTICALES Y LA NUEVA POLITICA TERRITORIAL VERTICAL}

La base económica de buena parte de la montaña pirenaica es terciario-turística sustituyendo paulatinamente la base primaria tradicional. Ha habido una mutación de funciones al avanzar la era industrial hacia la postindustrial (o neoindustrial) y adaptarse a las nuevas demandas de las sociedades urbanas periféricas, en las que el ocio se ha convertido en un negocio.

El cuadro natural pirenaico presenta una serie de "fragilidades" de cara a la conservación, ante la agresividad de las tecnologías duras con las que se aborda en ocasiones el negocio turístico, pero al mismo tiempo cuenta con una riqueza paisajística de gran atractivo y rentabilidad.

Desde el punto de vista de los nuevos desequilibrios verticales provocados por la actividad turística hay que diferenciar entre el turismo blanco de esquí, que suele ser el más agresivo, del turismo verde y rural.

La construcción de estaciones de esquí, de urbanizaciones a pié de pista y de carreteras que conduzcan a las mismas -como ha sucedido en Astún- supone una movilidad de tierras, una pérdida de vegetación, una incentivación de procesos erosivos, un deterioro paisajístico, una contaminación de las aguas, etc. Pero por otro lado hay que tener en cuenta que la única forma de crear empleo, mantener población residente e incluso acrecentarla, como en Saint-Lary, es a partir del turismo.

Por otro lado no siempre las estaciones de esquí van contra el ecosistema antrópico preindustrial, sino que con una sabia combinación, como la practicada por el alcalde de Saint-Lary, se puede volver al pastoreo para mantener el prado en las pistas.

De cualquier forma no hay que olvidar que turismo blanco, verde o rural se va a seguir dando en el Pirineo y los visitantes agreden el paisaje (incendios, basuras, etc.) si no hay una población residente que lo defienda y ésta existe en la medida en que haya puestos de trabajo rentable que principalmente aporta el turismo blanco.

La política de ordenación del territorio pirenaico debe compatibilizar un desarrollo económico sostenible, que básicamente se apoye en el turismo, con la mejora de la calidad de vida de la población residente y el respeto y recuperación del patrimonio natural y cultural.

La ordenación del territorio pirenaico implica clasificar y ordenar los usos del suelo que compiten ahora mismo en el Pirineo: uso residencial, agrícola, pecuario, 
forestal, industrial y turístico,... Debe clasificar y proteger los espacios naturales, recuperar y restaurar el medio contaminado. También debe atender a la protección del patrimonio etnológico y cultural, teniendo en cuenta que tanto el patrimonio natural como el cultural son el mejor activo con que cuenta el Pirineo para la actividad turística, base económica del Pirineo.

\section{EL MERCADO UNICO Y LA POLITICA TERRITORIAL HORIZONTAL}

El Mercado Unico abre nuevas perspectivas para la cooperación transfronteriza. La política de las Comunidades Europeas tiende a eliminar los efectos frontera entre las regiones mediante programas de apoyo transfronterizo como el INTERREG y creando instrumentos de gestión y ordenación conjuntos, pero para ello hay que superar las inercias plateadas en el territorio por las políticas anteriores, desequilibradoras en sentido N-S y E-W, volviendo de algún modo a la concepción integrada del espacio pirenaico.

El ejemplo de las facerías internacionales de pastos invitan a crear el Parque Internacional de los Pirineos o facerías de la nieve para la explotación del turismo blanco o la presentación de una candidatura binacional para la celebración de las Olimpiadas de invierno (con base en Jaca y Pau, por ejemplo) si se repite la candidatura de Jaca 98 en el futuro.

La existencia de caminos transpirenaicos, como el medieval de Santiago, sugiere la creación ejes Norte-Sur como la autovía Sagunto-Zaragoza-Somport-Pau-Burdeos, que, respetando el medio ambiente, con soluciones como la reducción de carriles o la plataforma rodante por los puntos más frágiles, suponga un nexo directo entre las arcos mediterráneo y atlántico de la geoeconomía europea. El espacio pirenaico ganaría clientela turística y también dada su calidad ambiental permitiría instalarse industrias de alta tecnología y no contaminantes en las pequeñas ciudades pirenaicas como subcontratadas de las grandes empresas instaladas en las grandes metrópolis periféricas (Zaragoza-G.M., Barcelona, Toulouse, etc.).

La autovía anterior y otras intrapirenaicas, así como las ferroviarias, entre las que hay que citar en primer lugar la reapertura del ferrocarril del Canfranc-Oloron, favorecerían el desarrollo de la red urbana intrapienaica a partir de las grandes metrópolis periféricas, muchas de las cuales se encuentran ya aglutinadas desde hace tres años en la red $C_{6}$, que comprende Barcelona, Valencia, Zaragoza, Montpellier, Toulouse y Palma. La iniciativa político-municipal trató de sumarse a una realidad económica de cooperación bastante consolidada por parte de las empresas privadas del área. Las pequeñas ciudades pirenaicas, pertenecientes hasta ahora a la periferia 
económica de los sistemas interurbanos liderados por Barcelona, Zaragoza o Toulouse, conectarían más entre sí dando lugar a un sistema transpirenaico de ciudades, que sólo aparece apuntado hoy en día en los extremos catalán y vasco.

Estos son algunos ejemplos de lo que tendrá que hacerse en el futuro para apoyarse en la sinergia que supone una política pirenaica integrada y abierta a los grandes ejes europeos de desarrollo, en la que se pueda apoyar el propio desarrollo local, dentro de las pautas del desarrollo sostenible que permita disminuir los desequilibrios verticales $\mathrm{y}$ horizontales del territorio pirenaico.

\section{BIBLIOGRAFIA}

ANGLADA, S.; BALCELLS, E.; CREUS, J.; GARCIA RUIZ, J.M.; MARTIN BONO, C.E. y PUIGDEFABREGAS, J. (1980): La vida rural en la montaña española (orientaciones para su promoción), Instituto de Estudios Pirenaicos, 107, 133 p., Jaca.

ASSO, I. (1798): Historia de la Economía Política de Aragón, Eston de Estudios Pirenaicos, 1947, Zaragoza, C.S.I.C.

AYERBE, J.M. (1908): Estudio crítico de los trabajos hidrológico-forestales realizados en la cuenca de cabecera del Aragón, Ministerio de Agricultura, Madrid.

BARRERE, P. (1952): "Types d'organisation des terroirs en Haut-Aragon", Actas I Cong. Int. de Est. Pirenaicos, Sec. IV, 5, 249-268, Zaragoza.

BIELZA DE ORY, V. y DALLA-ROSSA, G. (1988): Las relaciones socioeconómicas transpirenaicas. Colección "Cosas Nuestras", Instituto de Estudios Altoaragoneses, Huesca.

BIELZA DE ORY, V. y VALENZUELA, (1983): "Aspectos histórico-geográficos de la degradación de un ecosistema de Montaña: los altos valles pirenaicos aragoneses occidentales", Actas VII Cong. Int. de Estudios Pirenaicos, Jaca, 157-170.

BIELZA, V.; CORRAL, J.L.; ESCOLANO, S.; LALIENA, C.; SESMA, A. y UBIETO, A. (1986): Estudio histórico geográfico del Valle del Bielsa (Huesca), Instituto de Estudios Altoaragoneses, Huesca.

BRIVES, A. (1984): Pyrénées sans frontière: La vallée de Barèges et l'Espagne de XVIII siècle à nos jours., Société d'Etudes des Sep Vallées, Argelès-Gazost.

CAVAILLES, H. (1910): "Une fédération pyrénéene sous l'Ancien Regime. Les Traites de Lies et Passeries", Revue Historique, sep-déc., tomo 5, 1-34 y 241-276. 
CHAUVELIER, F. (1987): Reboisements et amenagement de l'espace: l'exemple de la province de Huesca, These de Doctorat, Univ. de Burdeaux III.

DATAR (1987): Les pyrénées, zone de massif, Toulouse.

DAUMAS, M. (1976): La vie rurale dans le Haut Aragon Oriental, C.S.I.C. Instituto de Estudios Oscenses e Instituto de Geografía Aplicada.

DESCHEEMAEKER, L. (1945):Une frontière inconnue, les Pyrénées de l'Ocean à l'Aragon, Université de Paris.

ESTEBANEZ, J. (1968): "Estudio de un municipio del Pirineo Central", Ap. española al XXI Congreso Geográfico Internacional, Madrid.

FAIREN-GUILLEN, V. (1961-62): "Notas para el estudio de las facerías internacionales pirenaicas", Pirineos, 59-66, 145-164, Zaragoza.

FAUCHER, D. (1951): Les Pyrénées françaises, Ed Larousse, Paris.

FLORISTAN, F. (1986): "Reflexión geográfica sobre las facerías de Navarra", Lies et passeries dans les Pyréneées, 123-139, Tarbes.

GARCIA RUIZ, J.M. (1976): "Modos de vida y niveles de renta en el Prepirineo del Alto Aragón Occidental", Est. Pirenaicos, 106.

GORRIA, A.J. (1986): "Algunos aspectos sobre los tratados de facerías entre los valles de Ansó y Aspe", Lies et passeries dans les Pyrénées, Tarbes.

GORRIA, A.J. (1993): El Pirineo Central como espacio frontera, 544 p., Tesis Doctoral, Universidad de Zaragoza.

LABOIRE, J.P. (1989): "El marco institucional de las políticas de montaña en Francia", Estudios Territoriales, 29, MOPU, Madrid.

LASANTA, T. (1989): "Evolución reciente de la agricultura de montaña", Geoforma Ediciones, Logroño.

LEFEBVRE, Th. (1933): Les modes de vie dans les Pyrénées atlantiques orientales, Colin, Paris.

LOSCERTALES, B. (1993): Jacetania, de espacio agrario a espacio turístico, Ed. Prames, 319 p., Zaragoza.

MALUQUER DE MOTES, J. (1966): "Consideraciones sobre el problema de la formación de los vasco", Problema de la prehistoria y de la etnología vasca, Inst. P. de Viana, Pamplona. 
MONTSERRAT RECODER, P. (1964): Ecología del pasto. Publicaciones del Centro Pirenaico de Biología, 68 p., Jaca.

MOPU-DATAR (1989): Actas del coloquio: Los Pirineos, montaña de Europa, Jaca 22-23 de junio de 1989, Madrid.

PAPY, M. (1986): "Mutilation d'un rite: la junte de Roncal et Baretous et la crise de nationalisme française dans les années 1890", Lies et passeries dans les Pyrénées, 197-233, Tarbes.

SERMET, J. (1960): "Comunications pyrénées et transpyrénéenns", Actas del Segundo Congreso Internacional de Estudios Pirenaicos, Luchon-Pau.

SERMET, J. (1983): La frontière des Pyrénées, Les amis du livre, Pyrénées, Pau.

SOLE SABARIS, L. (1951): Los Pirineos: el mundo y los hombres, Ed. Alberto Martin, Barcelona.

VIERS, G. (1973): Los Pirineos, Oikos-tau, Barcelona. 